\title{
COMPORTAMIENTO POBLACIONAL DEL ÁCARO Steneotarsonemus spinki EN VARIEDADES DE ARROZ UTILIZADAS EN COSTA RICA
}

\author{
Ruth León González' \\ RESUMEN
}

Comportamiento poblacional del ácaro Steneotarsonemus spinki, en variedades de arroz utilizadas en Costa Rica. El objetivo de este estudio fue determinar la preferencia del ácaro S. spinki en variedades de arroz bajo secano favorecido, el cual se realizó en la localidad Olla Cero, Palmar de Osa, Puntarenas, Costa Rica de junio a diciembre del 2005. Se utilizó un diseño de bloques completos al azar con 16 variedades y tres repeticiones, en parcelas de $10 \mathrm{~m}^{2}(2$ x $5 \mathrm{~m}$ ), con una separación de $1 \mathrm{~m}$ entre bloques. Se seleccionaron tres plantas al azar, y de cada una se evaluaron tres tallos, y de estos se tomaron tres vainas. Variables evaluadas: total de ácaros (huevos, inmaduros (larvas-ninfas) y adultos (hembras- machos) por variedad; los datos se sometieron a análisis de varianza y separación de medias según la prueba de Tukey al $5 \%$, además área bajo la curva $(A B C)$ del total de los ácaros. Las variedades se agruparon con base a la preferencia del ácaro. El grupo I, las menos preferidas: Palmar 18 (29), INTA 05 (570), Fedearroz-50 (1116), Senumisa 3 (1440), CR-4477 (1745) y Senumisa 2 (1777), grupo II, medianamente preferidas: CFX-18 (2643), CR-1821 (3598) y CR-5272 (4385) y grupo III, susceptibles al ácaro: CR-4102 (10449), Tempisque (12641), CR-1113 (13355), CR-4338 (15882), Setesa 9 (16301), INTA 04 (19901) y Senumisa 4 (21685). El mayor rendimiento lo obtuvo la variedad CR-5272 seguida de la variedad CFX-18 las cuales fueron medianamente preferidas. Por lo tanto no se presentó una relación directa entre las poblaciones del ácaro y los rendimientos del cultivo.

Palabras claves: preferencia, arroz secano, etapa fenológica, índice de producción.

\section{INTRODUCCIÓN}

El ácaro del vaneo del arroz Steneotarsonemus spinki Smiley, 1967, está ampliamente distribuido en Asia, ha ocasionado pérdidas en los rendimientos de un $60 \%$ por primera vez en China, a mediados de la década del setenta (INISAV, citado por Almaguel et al. 2004). En 1977 causó severos daños en Taiwán (Cheng y Hsiao, citado por Almaguel et al. 2004), y desde 1985 ha sido considerado como plaga del arroz para el Asia tropical (Almaguel et al. 2004). En Cuba se detectó a finales de 1997, (Ramos y Rodríguez 2003) y causó pérdidas hasta un $80 \%$ (Reyes 2005). Además, se encuentra en República Dominicana, Haití, Colombia, Panamá, Centroamérica, México, Belice y Estados Unidos (Hummela et al. 2009).

En Costa Rica en el año 2003, se observaron las primeras sintomatologías del manchado y vaneo del grano en la provincia de Guanacaste en aproximadamente 15000 hectáreas. Se atribuyó este problema a bacteriosis asociado a un efecto climático (alta temperatura y precipitación), luego esta sintomatología se extendió a todas las regiones arroceras del país y fue identificado en Bagaces, Guanacaste en el año de 2004.

La mayor incidencia del ácaro se favorece por altas temperaturas, disminución de las lluvias, dosis elevadas de fertilizantes nitrogenados y por un mal manejo general de la fertilización (Almaguel et al. 2003, Botta et al. 2003 y Miranda et al. 2003).

EI S. spinkiocasiona dos tipos de daño: directo, al alimentarse extrae el contenido de la vaina de las hojas lo que induce la deshidratación y la muerte del tejido, se alimenta de los granos en formación, impide su llenado (vaneo), se observa necrosis, marchitez y deformación en el raquis de la panícula en el momento

${ }^{1}$ Instituto Nacional de Innovación y Transferencia en Tecnología Agropecuaria, INTA. Costa Rica. nbonilla@ inta.go.cr 
de la floración; lesiones directas sobre el órgano floral y el grano en formación; atrofia y bloqueo del crecimiento. Indirecto: durante su alimentación, el ácaro inyecta sustancias tóxicas a las células vegetales para mantener el contenido celular fluyendo sin obstáculos; estas toxinas provocan deformaciones en el tejido vegetal, induce a la deformación del grano lo que se conoce como "pico de lora", además, Almaguel et al. (2003) indican que este ácaro transporta las esporas del hongo Acrocylindrium, Sarocladium oryzae (Sawada) Gams \& Hawksw, el cual causa manchado y pudrición de la vaina y la espiga. Más recientemente se relaciona con las bacterias Pseudomonas fuscovaginae y Burkolderia glumae.

Santos et al. (2001), indican que este ácaro es una plaga específica del cultivo del arroz, mientras que en el IDIAP-Panamá (2006) se menciona que esta plaga afecta los rendimientos del cultivo dependiendo de la susceptibilidad de la variedad, estas aseveraciones como muchas otras confirman que este ácaro, tiene preferencia varietal.

Estudios preliminares realizados en el INTA (León 2004 y León 2008), muestran variedades comerciales y de fundación con algún grado de tolerancia al ácaro al alcanzar considerables poblaciones por planta durante su ciclo fenológico, situación que podría ser utilizada en el manejo integrado del ácaro. Esta investigación de preferencia de $\mathcal{S}$. spinki por los materiales de arroz es importante para tomar decisiones en el momento de hacer liberaciones de variedades comerciales, generalmente los ácaros fitófagos tienen preferencia por un tipo de tejido vegetal en particular, siendo la razón principal por la cual se convierten en plagas que se localizan en determinadas partes de las plantas durante su ciclo de vida.

El objetivo de este estudio fue determinar la preferencia del ácaro $\mathcal{S}$. spinki en 16 variedades de arroz bajo secano favorecido utilizadas en Costa Rica.

\section{MATERIALES Y MÉTODOS}

El estudio se realizó en Finca 18, localidad Olla Cero, Palmar Norte de Osa, Puntarenas, durante los meses de junio a diciembre del 2005. La temperatura media anual osciló en un rango de $25^{\circ} \mathrm{C}$ y $30{ }^{\circ} \mathrm{C}$. La precipitación promedio del año 2005 en el lugar del estudio fue de $4479,29 \mathrm{~mm}$ y la precipitación promedio desde el mes de junio a diciembre fue de $487,79 \mathrm{~mm}$, la mayor en el mes de octubre $(896,62 \mathrm{~mm})$ y la mínima en diciembre $(40,64$ $\mathrm{mm}$ ). La precipitación promedio anual en los últimos cinco años en la zona de estudio ha sido de $3105,08 \mathrm{~mm}$.

La precipitación promedio del año 2005 en el lugar del estudio fue de $4479,29 \mathrm{~mm}$ y la precipitación promedio desde el mes de junio a diciembre fue $487,79 \mathrm{~mm}$ dándose la mayor precipitación en el mes de octubre $(896,62)$ y la mínima en el mes de diciembre $(40,64 \mathrm{~mm})$ (Figura 1).

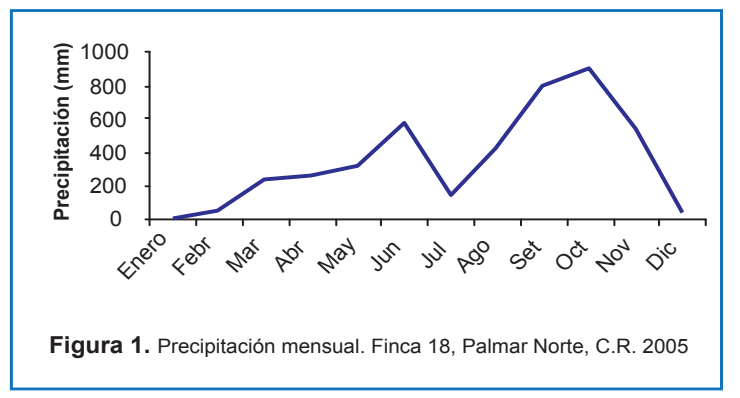

Se empleó un diseño de bloques completos al azar, con tres repeticiones, la parcela experimental fue de $10 \mathrm{~m}^{2}(2 \times 5 \mathrm{~m})$, con una separación de $1 \mathrm{~m}$ entre bloques. Se evaluaron 16 variedades de arroz (Cuadro $1)$, de las cuales al momento de hacer el estudio 12 eran comerciales en Costa Rica: CR-5272, CR-1821, CR-4477, FEDEARROZ 50, SENUMISA-04, CR-4106, CR-1113 y más recientemente CFX18, SETESA 9, SENUMISA 02, SENUMISA 03 y Palmar 18.

La finalidad de conocer las características de cada variedad fue tratar de encontrar respuesta a las preferencias del ácaro (estructura, color, disposición de las vainas, entre otros) a la variedad, evaluadas, bajo el sistema de secano favorecido (Cuadro 1). 
Cuadro 1. Características de las variables evaluadas. Palmar Norte, CR. 2005.

\begin{tabular}{|c|c|}
\hline Variedad & Características \\
\hline CFX-18 & $\begin{array}{l}\text { Es una variedad adaptada a secano, de macollamiento mediano, porte bajo, ciclo } \\
\text { precoz, de } 95 \text { a } 100 \text { días después de la germinación (ddg). }\end{array}$ \\
\hline CR-4102 & $\begin{array}{l}\text { Su ciclo se considera precoz ya que lo cumple entre los } 105-110 \text { ddg, alcanza la } \\
\text { floración entre los } 75 \text { y } 80 \text { días }\end{array}$ \\
\hline Palmar 18 & $\begin{array}{l}\text { Material de ciclo corto o precoz, con variaciones de } 105 \text { a } 115 \text { días de germinación } \\
\text { a cosecha, el inicio de formación de primordio floral se da entre los } 48 \text { y } 55 \text { días. } \\
\text { Inicia floración entre } 75-80 \text { días, maduración a los } 105-115 \text { días. Posee alta } \\
\text { adaptabilidad a zonas de siembra de secano. De macollamiento alto, tolerante al } \\
\text { acame, altura de planta } 105-110 \mathrm{~cm} \text {. }\end{array}$ \\
\hline CR-5272 & $\begin{array}{l}\text { Porte bajo, hojas erectas, macollamiento moderado, resistente al acame, con } \\
\text { floración entre los } 80 \text { y } 85 \text { días después de la siembra y una duración a la cosecha } \\
\text { entre } 110 \text { y } 115 \mathrm{ddg} \text {. }\end{array}$ \\
\hline CR-4477 & $\begin{array}{l}\text { Es de macollamiento intermedio, en estudios previos ha mostrado ser menos } \\
\text { preferida por el ácaro del vaneo. Esta variedad es de ciclo intermedio (115-120 } \\
\text { días), florece a los } 85 \text { días, tolerante al volcamiento. }\end{array}$ \\
\hline Tempisque (IW7) & $\begin{array}{l}\text { Línea de ciclo cortó con } 115 \text { días a cosecha. Esta línea tiene la característica de } \\
\text { contar con el gen de resistencia a las Imidazolinonas. }\end{array}$ \\
\hline Senumisa 2 & $\begin{array}{l}\text { Su macollamiento es intermedio. Es de ciclo intermedio, ya que completa su ciclo } \\
\text { a los } 115 \text { días y florece a los } 85 \mathrm{ddg} \text {. }\end{array}$ \\
\hline Senumisa 3 & $\begin{array}{l}\text { De macollamiento intermedio. Se considera de ciclo precoz ya que inicia } \\
\text { macollamiento entre los } 30-35 \mathrm{ddg} \text {, floración entre } 80-85 \text {. }\end{array}$ \\
\hline Senumisa 4 & $\begin{array}{l}\text { Es de ciclo precoz, inicia macollamiento a los 30-35 ddg y la floración a los 85-95 } \\
\text { ddg. }\end{array}$ \\
\hline SETESA 9 & $\begin{array}{l}\text { Esta variedad es de hojas erectas, color de lámina verde intenso, con tonalidad } \\
\text { púrpura bajo ciertas condiciones. Tienen una altura de planta de } 94 \mathrm{~cm} \text {, florece } \\
\text { a los } 97 \text { días, la cosecha se da a los } 128 \text { días, el macollamiento es intermedio o } \\
\text { moderado susceptible al volcamiento. }\end{array}$ \\
\hline Fedearroz -50 & $\begin{array}{l}\text { Variedad de ciclo intermedio a tardío (125-128 ddg) y florece a los } 95 \text { días. } \\
\text { Tolerante al acame. Su macollamiento es intermedio. }\end{array}$ \\
\hline CR-1113 & $\begin{array}{l}\text { Paja corta, hojas erectas, tallos gruesos, buen macollamiento, resistente al } \\
\text { acame, florece entre } 95 \text { y } 100 \text { días después de la siembra (dds), se cosecha a } \\
\text { los } 127 \text { y } 135 \text { días. }\end{array}$ \\
\hline CR-1821 & $\begin{array}{l}\text { Tiene gran capacidad de macollamiento y resistencia al acame. Sus hojas se } \\
\text { caracterizan por ser de crecimiento laxas y erectas; florece entre los } 95 \text { y } 100 \\
\text { días y se cosecha entre los } 125 \text { y } 130 \text { días. }\end{array}$ \\
\hline INTA 04 & $\begin{array}{l}85 \text { días a floración, } 115 \text { días a cosecha, macollamiento medio, tolerante al acame. } \\
\text { Susceptible al ácaro } \mathcal{S} \text {. spinki, }\end{array}$ \\
\hline INTA 05 & $\begin{array}{l}88 \text { días a floración, } 120 \text { días a cosecha, macollamiento alto, tolerante al acame y } \\
\text { al ácaro } \mathcal{S} \text {. spinki. }\end{array}$ \\
\hline CR-43 & $\begin{array}{l}\text { El tipo de macollamiento es intermedio, resistente al volcamiento, florece a los } 87 \\
\text { días, su ciclo a cosecha lo completa en } 118 \text { días. }\end{array}$ \\
\hline
\end{tabular}

Fuente: MAG, ONS, INTA.

En el Cuadro 2 se incorporó la designación y los progenitores de cada variedad, así como la genealogía, cruce, año en que fue registrada y la institución registrante. 
Cuadro 2. Descripción de las variedades evaluados. Palmar Norte, CR. 2005.

\begin{tabular}{|c|c|c|c|c|}
\hline Variedad & $\begin{array}{l}\text { Institución } \\
\text { registrante }\end{array}$ & Año & Genealogía & Cruce \\
\hline CFX-18 & INARROZ & 2000 & LA2207 & L-202/Lemont \\
\hline CR-4102 & $\begin{array}{l}\text { MAG-ONS- } \\
\text { A C P S - } \\
\text { OFIARROZ }\end{array}$ & 1999 & CT10825-1-2-1-1-1 & $\begin{array}{l}\text { CT6516-23-10-1-2-2/CT6750-9-2- } \\
\text { 4-M-1-M-1 }\end{array}$ \\
\hline Palmar 18 & SENUMISA & 2007 & $\begin{array}{l}\text { FL03187-12P-5- } \\
\text { 2P-3P-M }\end{array}$ & $\begin{array}{l}\text { F L } 03160 \text { - } 6 \text { P - } 12 \text { - } 2 \text { P - } 1 \text { P - M } \\
\text { (ORIZYCA1/IR21015-72-3-3-3-1// } \\
\text { CT10310-15-3-2P-4-3) }\end{array}$ \\
\hline CR-5272 & MAG & 1975 & P 753-19-1-1-CR29 & IR930-80/IR822-432 \\
\hline CR-4477 & $\begin{array}{l}\text { MAG-ONS- } \\
\text { OFIARROZ- } \\
\text { ACPS }\end{array}$ & 2003 & $\begin{array}{l}\text { C T } 11408-6-\mathrm{F} 4- \\
21 \mathrm{P}-2\end{array}$ & $\begin{array}{l}\text { P } 42277-\text { F } 2 \text { - } 2-9-1 \text { X I } \\
\text { COL1 XM312A-74-2-8-8/P3059- } \\
\text { F4-79-1-1B }\end{array}$ \\
\hline $\begin{array}{l}\text { Tempisque } \\
\text { (IW7) }\end{array}$ & INARROZ & $\begin{array}{l}\text { Sin } \\
\text { registrar }\end{array}$ & - & Parentales: \\
\hline Senumisa 2 & SENUMISA & 2004 & $\begin{array}{l}\text { CT10323-29-4-1- } \\
1 \mathrm{~T}-2 \mathrm{P}\end{array}$ & $\begin{array}{l}\text { P3084-F4-56-2-2/P3844-F3-19-1- } \\
\text { 1B-1X//CT8154-1-9-2. }\end{array}$ \\
\hline Senumisa 3 & SENUMISA & 2004 & & P5413-8-3-5-11/СT9145-4-15-1-1 \\
\hline Senumisa 4 & SENUMISA & 2005 & $\begin{array}{l}\text { СT8050/P5413-8- } \\
3-5-11-2 X\end{array}$ & $\begin{array}{l}\text { CT8050 como progenitor femenino } \\
\text { está conformado por el cruce de: } \\
\text { 18521/IR21015 y el masculino: } \\
\text { P5413-8-3-5-11-2X proviene } \\
\text { del cruce CR-1113/IRAT122// } \\
\text { Colombia 1/5685 }\end{array}$ \\
\hline SETESA 9 & CIAT & 1985 & $\begin{array}{l}\text { PNA } 2 \text { F6-1055-1-5 } \\
\text { P4034F5-21 }\end{array}$ & CICA8//CICA4/Componi \\
\hline $\begin{array}{l}\text { Fede arroz } \\
-50\end{array}$ & SENUMISA & 2001 & FB0007-3-1-6-1-M & $\begin{array}{l}\text { ORYZICA LLANOS } \\
\text { 1274-6-8M-1-3M-1 }\end{array}$ \\
\hline CR-1113 & MAG & 1974 & IR822-81-CR2 & IR 8//PANKHARI 203/IR 8 \\
\hline CR-1821 & MAG & 1985 & $\begin{array}{l}\text { P 881-19-22-4-1- } \\
\text { 1B-1B-CR1-61 }\end{array}$ & IR 22//IR930-147-8/COLOMBIA 1 \\
\hline INTA 04 & INTA-CR & 2005 & CT18685-7-1-3-2 & - \\
\hline INTA 05 & INTA-CR & 2005 & CT18657-12-4-21-1 & СТ8628/P9743-F2-85-6-1X \\
\hline CR-4338 & $\begin{array}{l}\text { MAG-ONS- } \\
\text { OFIARROZ- } \\
\text { ACPS }\end{array}$ & 1999 & СТ8665-1-1-1P-4 & $\begin{array}{l}\text { P } 4277-\text { F } 2-2-9-1 \text { X I } \\
\text { COL1XM312A-74-2-8-8/P3059- } \\
\text { F4-79-1-1B }\end{array}$ \\
\hline
\end{tabular}

Fuente: INTA, ONS, SENUMISA 


\section{Muestreos de ácaros}

Los muestreos se realizaron a partir de los 41 hasta los $104 \mathrm{ddg}$, de manera tal que se efectuaron siete evaluaciones, cada variedad se encontraba en distinta fase fenológica. Para ejecutar la evaluación se tomó al azar de cada parcela tres tallos en cada fecha de conteo, para un total de 84 tallos por tratamiento, los cuales se trasladaron en bolsas plásticas a $500 \mathrm{~m}$ de donde se tomo la muestra al lugar del conteo. Las vainas se cortaron con un bisturí a la altura del punto de inserción entre la hoja y la vaina que corresponde a la lígula. El conteo se realizó de forma manual y directa en estereoscopio con luz arriba y abajo y hasta $40 \times$ de aumento. Se registraron los diferentes estadios del ácaro huevos, inmaduros y adultos (hembras-machos).

\section{Labores agronómicas}

La densidad promedio de siembra fue de 250 plantas por parcela, lo cual correspondió a 138 gramos de semilla para $10 \mathrm{~m}^{2}$, equivalente a 140 $\mathrm{kg} / \mathrm{ha}$. La siembra se realizó al voleo. Durante todo el periodo de estudio no se aplicaron acaricidas. A excepción del insecticida a base de Etofenprox para disminuir las poblaciones del chinche de la espiga (Oebalus insularis) en la fase de estado lechoso donde se procuró tomar los tallos del último muestreo antes de la aplicación.

Las parcelas fueron tratadas según las prácticas habituales del manejo agronómico del cultivo, se fertilizó antes del inicio de la formación del primordio floral, a base de urea, cloruro de potasio, 18-46-0 y zinc foliar. En total se alcanzaron las siguientes cantidades de elementos por hectárea: nitrógeno $130 \mathrm{~kg}$, fósforo $40 \mathrm{~kg}$ y potasio $60 \mathrm{~kg}$. Para la protección contra enfermedades se aplicaron triazoles y carbendazina. Para controlar arvenses se utilizaron herbicidas hormonales, graminicidas y sulfunilureas.

${ }^{2}$ Laboratorio de Control de Calidad de CONARROZ 2009

\section{Índice de producción}

Para obtener el índice de producción se cosecharon 20 panículas en los $30 \mathrm{~m}^{2}$ de área cultivada para sacar el peso en 1000 granos, esta metodología se planteó debido a que durante el desarrollo del cultivo las condiciones climáticas fueron muy adversas (altas precipitaciones, sequía, vendavales) por lo que en algunas de las parcelas se perdieron plantas lo que hizo que no hubiera homogeneidad en elárea sembrada. El peso fue realizado en el Laboratorio de la Corporación Arrocera Nacional (CONARROZ) ${ }^{2}$

\section{Se evaluaron las siguientes variables}

1- Total de ácaros: huevos, inmaduros, adultos (hembras y machos) por variedad.

2- Rendimiento (peso de 1000 granos de arroz en granza en 30 metro cuadrados).

3- Área bajo la curva (ABC) del total de los ácaros.

4- Correlación de las poblaciones del ácaro frente la fase fenológica del cultivo.

Los datos obtenidos del conteo de los distintos estados del ácaro se sometieron a análisis de varianza y separación de medias según la prueba de Tukey al 5\% mediante el paquete estadístico SAS (Statistical Análisis Systems versión 6.11). Con base en el conteo total de ácaros se calculó el área bajo la curva $(A B C)$ mediante la fórmula: $A B C: \sum\left(\left(Y_{i}+Y_{i+1}\right) / 2\right)$ * $\left(T_{i+1}-T_{i}\right) T$ : tiempo y: ácaros.

Para construir las gráficas de comportamiento del ácaro se utilizó el total de ácaros (huevos, inmaduros y adultos) por variedad. Los cuales se calcularon en base al total de ácaros del área bajo la curva $(A B C)$ mediante la fórmula: ABC: $\sum\left(\left(Y_{i}+Y_{i+1}\right) / 2\right) *\left(T_{i+1}-T_{i}\right) T$ : tiempo y: ácaros.

El ABC consiste en determinar el comportamiento del ácaro con respecto a cada variedad a través del tiempo. Cada tratamiento se representa en un sistema de coordenadas en que la ordenada $(x)$ es muestreo y la 
abscisa (y) es la variable de respuesta. Se origina una línea de comportamiento que a su vez deja un área bajo ésta que se divide en secciones trapezoidales determinadas por la distancia entre muestreos y el comportamiento de la variable de respuesta en el período específico; la sumatoria de todas las áreas de las secciones trapezoidales (áreas parciales), resulta en el área total bajo la curva (Vargas 2006) $)^{3}$.

\section{RESULTADOS Y DISCUSIÓN}

\section{Análisis de las poblaciones de $\mathcal{S}$. spinki mediante el área bajo la curva $(A B C)$, usando el total de ácaros.}

Cuadro 3. Área bajo la curva $(\mathrm{ABC})$ de $\mathcal{S}$. spinkipor variedad registrados en el periodo de muestreo. Secano favorecido. Palmar Norte, CR. 2005.

\begin{tabular}{|l|l|}
\hline Variedad & Totales (Hembras, machos, inmaduros y huevos) \\
\hline CFX-18 & $2643(\mathrm{bc})^{*}$ \\
\hline CR-4102 & $10449(\mathrm{abc})$ \\
\hline Palmar 18 & $29(\mathrm{c})$ \\
\hline CR-5272 & $4385(\mathrm{bc})$ \\
\hline CR-4477 & $1745(\mathrm{c})$ \\
\hline Tempisque & $12641(\mathrm{abc})$ \\
\hline Senumisa 2 & $1777(\mathrm{c})$ \\
\hline Senumisa 3 & $1440(\mathrm{c})$ \\
\hline Senumisa 4 & $21685(\mathrm{a})$ \\
\hline Setesa 9 & $16301(\mathrm{ab})$ \\
\hline Fedearroz-50 & $1116(\mathrm{c})$ \\
\hline CR-1113 & $13355(\mathrm{abc})$ \\
\hline CR-1821 & $3598(\mathrm{bc})$ \\
\hline INTA 04 & $19901(\mathrm{a})$ \\
\hline INTA 05 & $570(\mathrm{c})$ \\
\hline CR-4338 & $15882(\mathrm{ab})$ \\
\hline
\end{tabular}

* Columnas con igual letra no difieren estadisticamente según pueba de Tukey al $5 \%$

\section{Grupo I. Variedades menos preferidas por el ácaro}

Este grupo se forma por las variedades que registraron menores cantidades poblacionales del ácaro durante el crecimiento de la planta, en el periodo comprendido de 41 a 104 ddg. Es decir desde la formación del primordio hasta cosecha. Dentro de este grupo se observaron poblaciones totales de ácaros de 29 a 1777 durante su ciclo fenológico. Entre estas variedades tenemos a Senumisa Hunsa conocida como Palmar 18 con (29 ácaros), seguida de INTA 05 (570 ácaros), Fedearroz-50 (1116 ácaros), Senumisa 3 (1440 ácaros), CR-4477 (1745 ácaros) y Senumisa 2 (1777 ácaros). La Figura 2 ilustra cada una de las variedades que formaron el grupo 1.

\footnotetext{
${ }^{3}$ Vargas, L. 2006. Área bajo la curva. Entrevista. INTA - Costa Rica. Comunicación personal.
} 


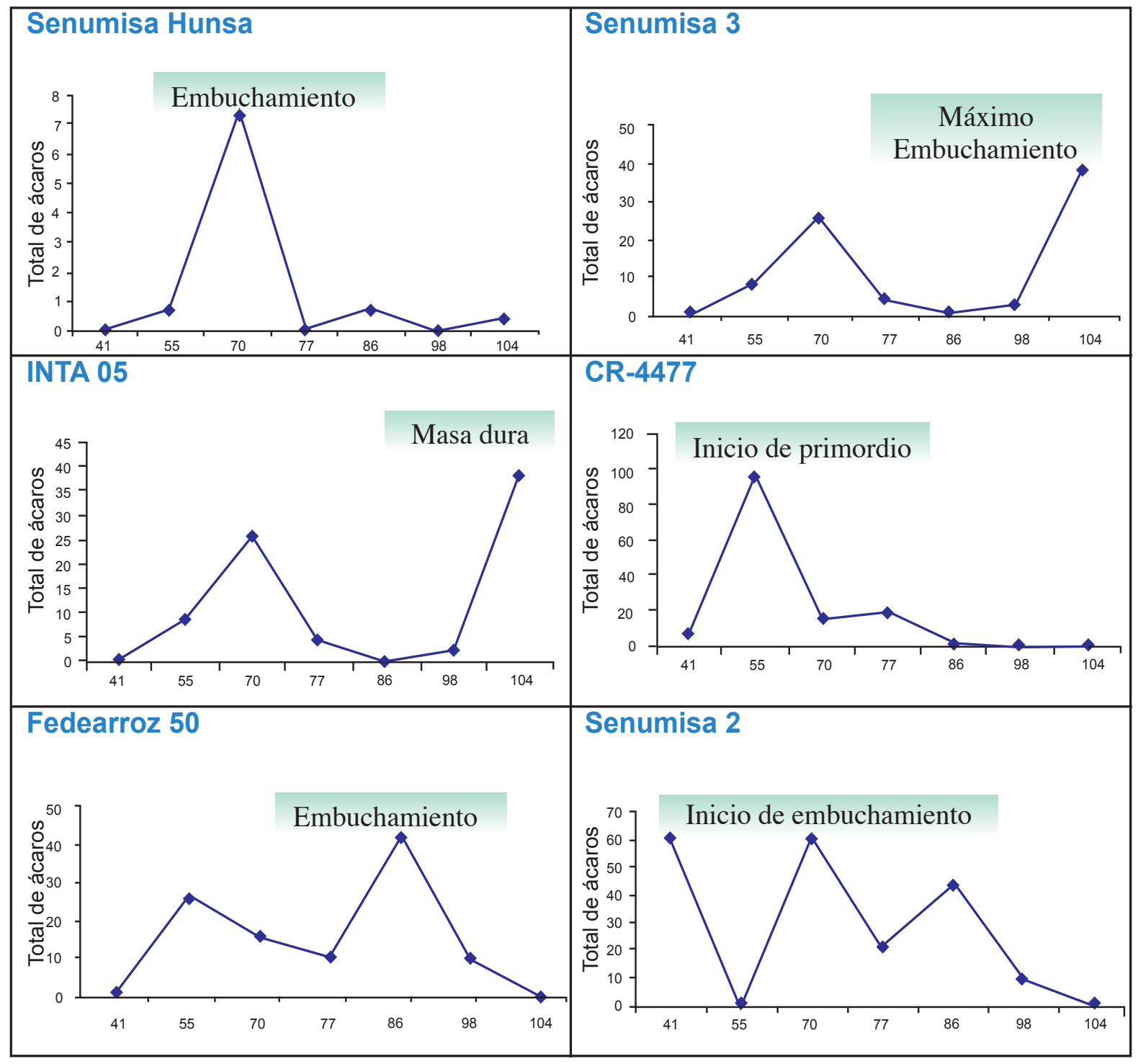

Figura 2. Área bajo la curva $(A B C)$ para totales (adultos, inmaduros y huevos) de $\mathcal{S}$. spinki por variedad. Palmar Norte, CR. 2005.

Palmar 18 presentó poblaciones promedio de 1,38 ácaros por planta, registrando una alza poblacional en la etapa de panzoneo (70 dds), pero en la edad de los 77 dds, que coincidió con la etapa fenológica de masa, se observó una disminución drástica de la población. Manteniendo poblaciones bajas hasta la cosecha. El resultado de uno de los componentes de rendimientos promedio de esta variedad se registró en 17,8 g/peso de 1000 granos.
INTA-05 registró poblaciones promedio de 27,14 ácaros/planta. Se observó alzas poblacionales a los $70 \mathrm{ddg}$ que corresponde a la etapa de panzoneo, luego cayeron las poblaciones hasta los $98 \mathrm{ddg}$ en la etapa de leche-masa, pero ocurrió una nueva alza poblacional al final de la maduración, lo que hace pensar en la importancia de realizar una desinfección del área a sembrar, y un buen manejo de los residuos de cosecha para que no existan fuentes de inóculo en el momento de la siembra. El rendimiento de esta variable fue de 17,8 g/peso de 1000 granos. 
Fedearroz 50 mostró una curva de población con cantidades superiores a 17,71 ácaros/tallo y el mayor pico poblacional lo alcanzó a los 86 dds, en la etapa fenológica de panzoneo, para luego descender hasta la cosecha, su rendimiento de campo fue de $14,9 \mathrm{~g} /$ peso de 1000 granos.

Senumisa 3 presentó 68,57 ácaros/tallo en promedio por planta durante su ciclo del cultivo. Las mayores poblaciones se presentaron en la etapa reproductiva, muy cercana a la floración, para luego aumentar hacia la maduración, esta situación debe considerarse por ser una fuente de inóculo para la siembra siguiente. El rendimiento registrado fue de $11,4 \mathrm{~g} /$ peso de 1000 granos.

Senumisa 2, en general presentó 84,62 ácaros/planta en promedio, presento una curva de población con fluctuaciones marcadas, inició desde el primer muestreo a los 41 días en la etapa de elongación del tallo con altas poblaciones, para luego descender a los 55 días en la etapa de primordio, a los 70 dds vuelve a aumentar las poblaciones similares a la obtenidas a los 41 dds, manteniendo esas poblaciones en su etapa reproductiva, hacia la maduración las poblaciones disminuyeron. El rendimiento fue de $15,0 \mathrm{~g} /$ peso de 1000 granos.

CR-4477 registró poblaciones medias de 83,1 ácaros/planta. En la etapa fenológica de inicio de primordio alcanzó el pico poblacional, después de esta etapa presentó una disminución de la población hacia la etapa de maduración y cosecha. El rendimiento cosechado fue de 14,4 g/peso de 1000 granos.

\section{Grupo II. Variedades medianamente preferidas}

Este grupo estuvo conformado por tres variedades que presentaron poblaciones entre los 2,643 y 4,385 como se muestra a continuación: CFX-18 (2643), CR-1821(3598) y CR-5272 (4385). Las dos primeras variedades obtuvieron los mayores rendimientos, pero la variedad CR-1821 fue la penúltima en cuanto al rendimiento como se muestra en el Figura 3.

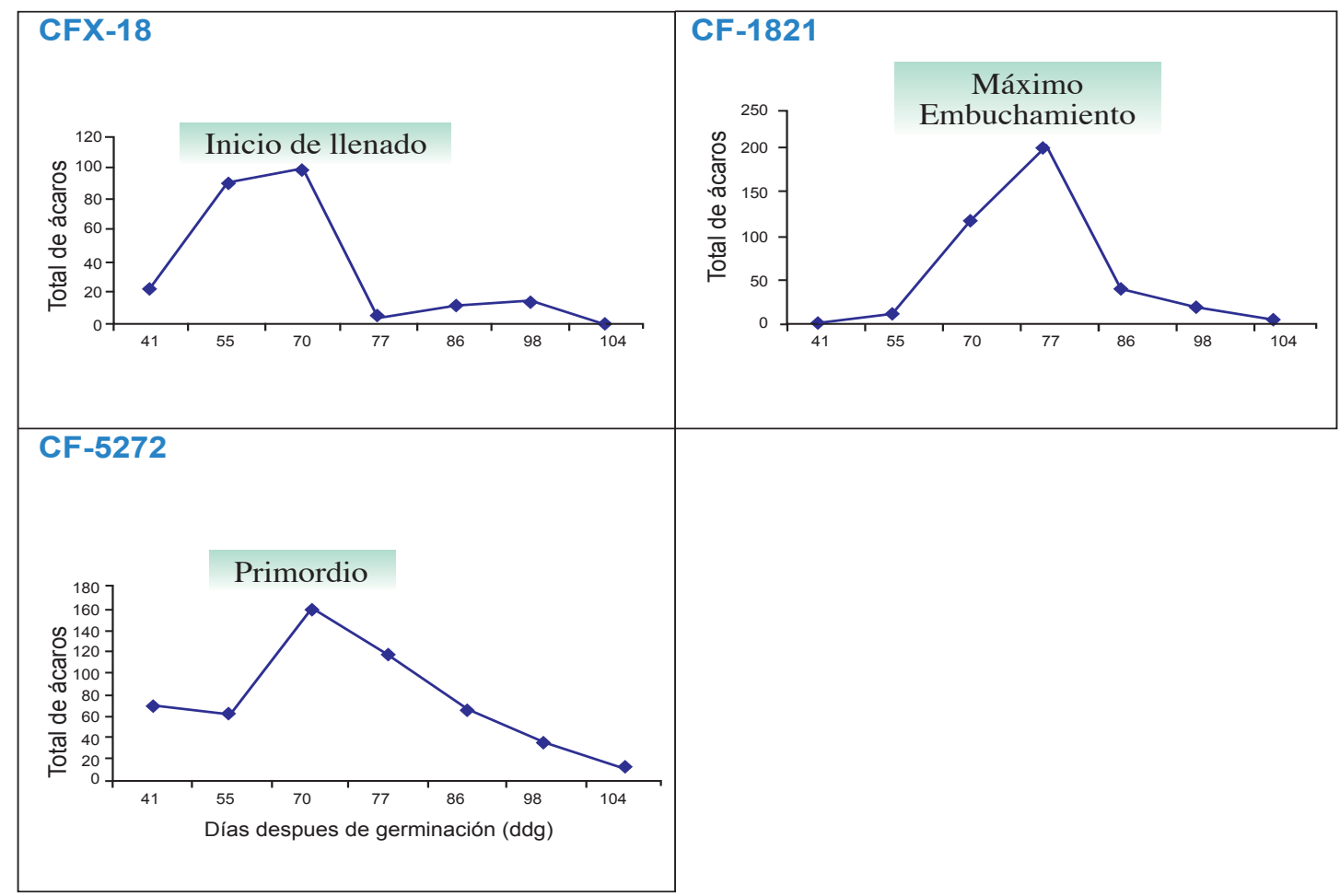

Figura 3. Área bajo la curva (ABC) para totales (adultos, inmaduros y huevos) de S. spinkipor variedad del grupo 2. Palmar Norte, CR. 2005. 
CFX-18 registró poblaciones medias de 125,86 ácaros/planta, las mayores poblaciones se observaron en la etapa vegetativa entre los 55 y 70 dds entre desarrollo de panícula y floración bajando la población en la etapa pastosa, mantuvo bajas las poblaciones hasta su cosecha. El rendimiento registrado fue 18,9 g/peso de 1000 granos.

CR-5272 presentó un promedio de 208,81 ácaros/planta, se observó poblaciones altas desde su etapa vegetativa, con altas poblaciones al momento de la floración, la disminución de la población se mostró hacia la maduración, pero en altas poblaciones. Su rendimiento fue 22,4 g/peso de 1000 granos.

CR-1821 en promedio alcanzó 172,33 ácaros/tallo, en esta variedad se observó un alza poblacional entre los 70 y 77 dds que correspondió a las etapas de primordio y previo a floración, disminuyendo gradualmente desde los 86 dds hasta la cosecha, el rendimiento registrado fue de $13,2 \mathrm{~g} /$ peso de 1000 granos.

\section{Grupo III. Variedades más preferidas por el ácaro}

La población total durante el ciclo fenológico de las diferentes variedades de este grupo osciló entre los 4,338 y 21,685 ácaros de sus diferentes estadios del ácaro. Esta población se muestra a continuación: CR-4102 (10 449), Tempisque (12 641), CR-1113 (13 355), CR-4338 (15 882), Setesa 9 (16 301), INTA 04 (19 901) y Senumisa 4 (21 685).

En la Figura 4 se muestran los diferentes gráficas poblacionales y se indica el estado fenológico de la planta en que alcanzó el pico poblacional.

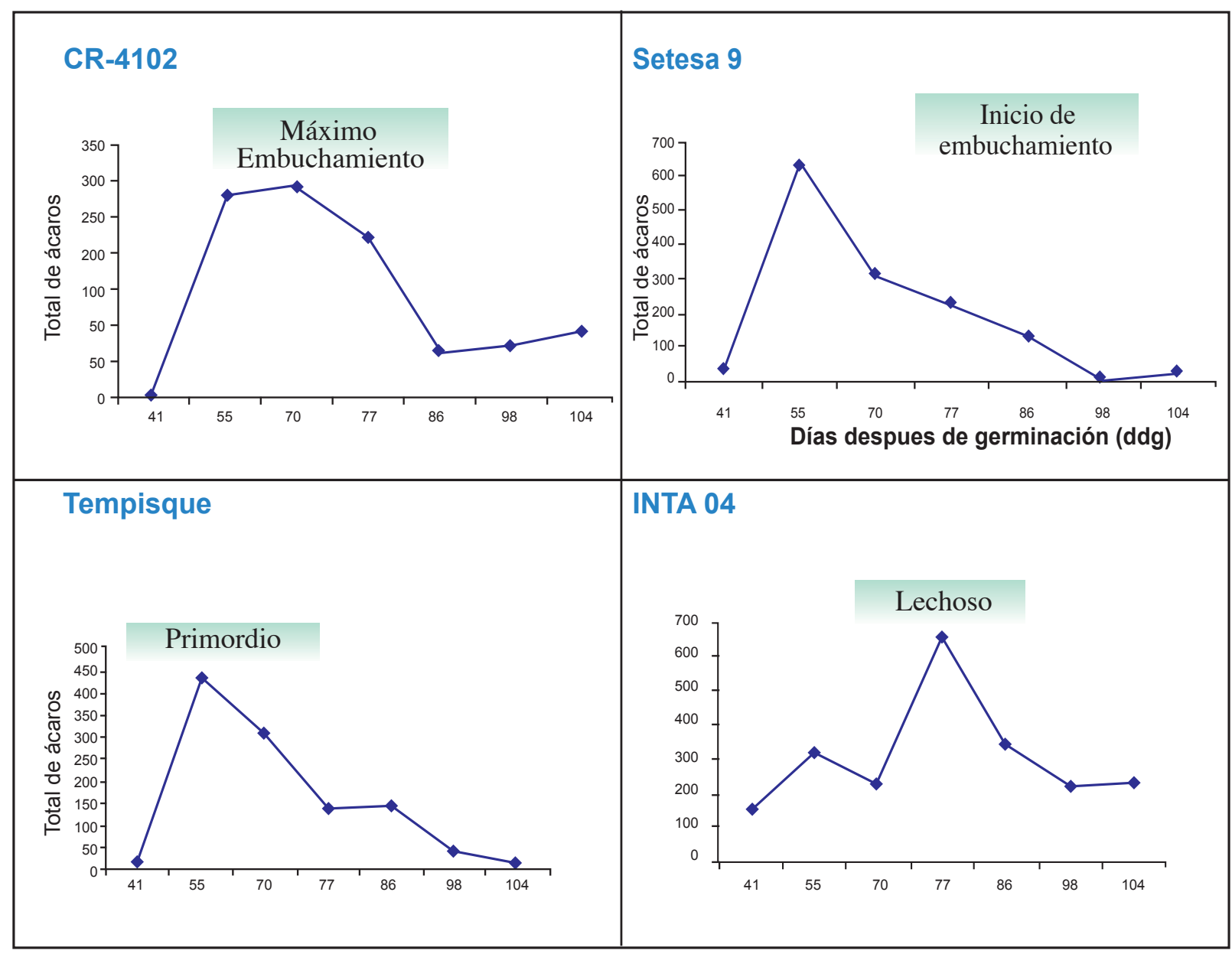




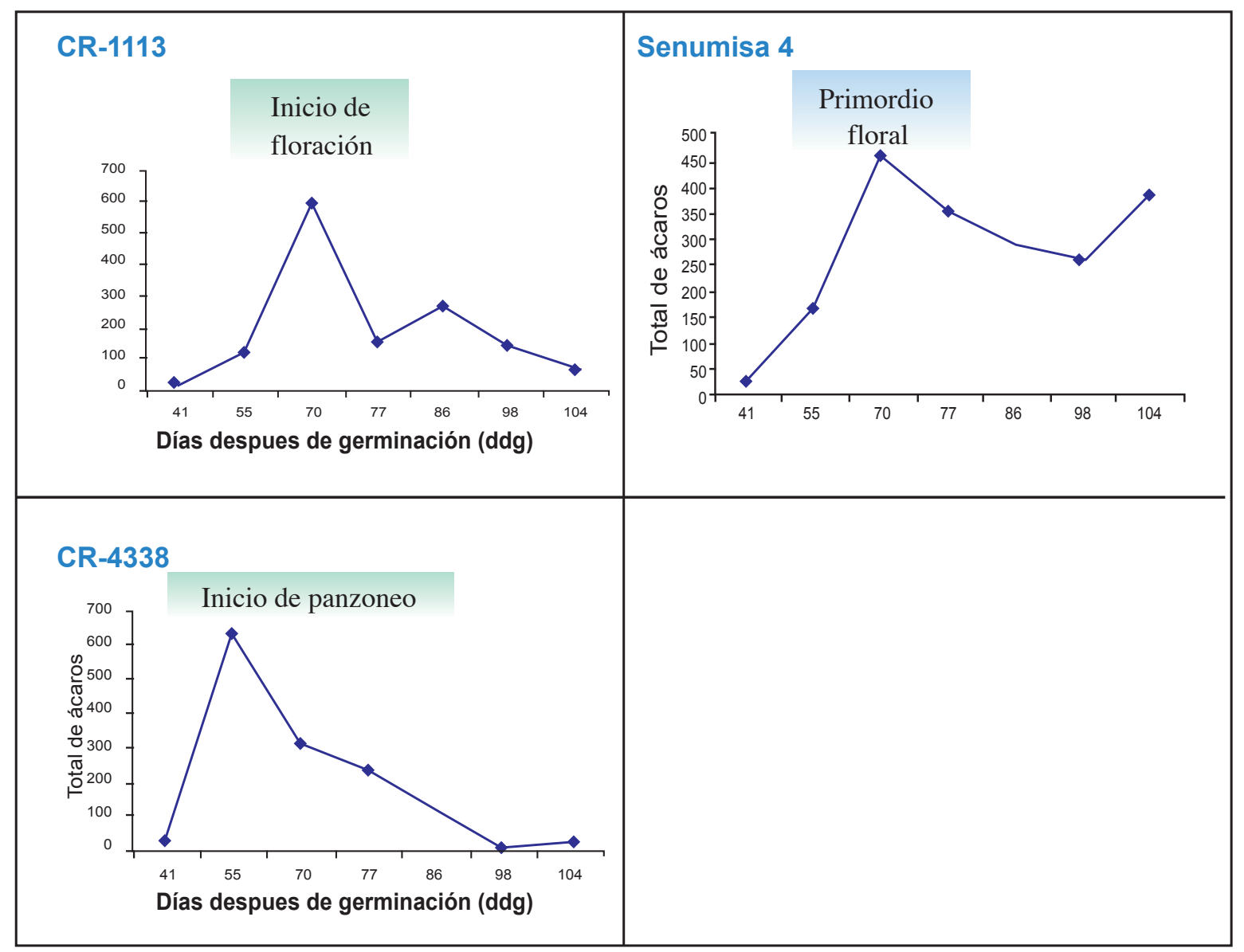

Figura 4. Área bajo la curva ( $A B C$ ) para totales (adultos, inmaduros y huevos) de S. spinki por variedad del grupo 3. Palmar Norte, CR. 2005.

CR-4102 esta variedad alcanzó un promedio de 497,57 ácaros/planta, consiguió el pico poblacional entre los 55 y 70 dds lo que correspondió a las etapas fenológicas de inicio de primordio y máximo panzoneo, disminuyendo levemente hacia la etapa de maduración y cosecha, sin embargo siempre mantuvo poblaciones altas en todo su ciclo fenológico. El rendimiento promedio fue de $\left(60,1 \mathrm{~g} / 30 \mathrm{~m}^{2}\right) 17,8 \mathrm{~g} /$ peso de 1000 granos.

SETESA 9 presentó un promedio de 776,24 ácaros/planta, alcanzando sus máximas poblaciones en la etapa de inicio de primordio, inició una disminución de las poblaciones desde la etapa de máximo panzoneo o previo a floración hasta el estado de leche, para aumentar de nuevo en la etapa de masaleche. El rendimiento fue de $16,7 \mathrm{~g} /$ peso de 1000 granos.
Tempisque (INARROZ, IW7) mostró un promedio de 602,14 ácaro/planta, las máximas poblaciones se alcanzaron en la etapa de primordio, manteniendo altas poblaciones en las fases vegetativa, reproductiva e inicios de maduración de hembras, machos e inmaduros. El rendimiento alcanzado fue de $14,6 \mathrm{~g} /$ peso de 1000 granos.

INTA 04 alcanzó un promedio de 947,67 ácaros/planta, a los 41 dds que correspondió a las etapas entre plántula y macollamiento, para alcanzar la mayor población a los 77 dds, etapa de leche, manteniendo poblaciones durante la fase maduración y cosecha. Esta variedad presentó durante la fase reproductiva altas poblaciones del ácaro. Su rendimiento fue de 14,4 g/peso de 1000 granos. 
CR-1113 presentó un promedio de 635,95 ácaros/planta, alcanzó las mayores cantidades de ácaros en las vainas en la fase reproductiva propiamente en la etapa de inicio de floración que correspondió a los 70 dds. En la etapa de elongación del tallo inicio a infestarse la planta de ácaros y hasta cosecha mantuvo altas las poblaciones. El rendimiento obtenido fue de $14,3 \mathrm{~g} /$ peso de 1000 granos.

Senumisa 4 el promedio de ácaros fue de $1036,62 /$ planta, iniciando a infestarse en la etapa de plántula (41 dds) con un promedio de 3,33 ácaros/vaina y aumentando las poblaciones hasta alcanzar el pico poblacional en la etapa de inicio de floración con 149,89 ácaros/planta, continuando con la etapa de cosecha con 125 ácaros/planta. El rendimiento de esta variedad fue de 13,9 g/peso de 1000 granos.
CR-4338 con un promedio de 756,29 ácaros/ planta, alcanzó el pico poblacional a los 55 días que corresponde a la etapa de inicio de panzoneo o desarrollo de panícula. El rendimiento de esta variedad fue de $14,1 \mathrm{~g} /$ peso de 1000 granos.

Recapitulando, en la Figura 5, vemos en orden ascendente como las variedades fueron infestadas por el ácaro y a pesar de esas diferencias las plantas no se vieron afectadas en su vigor, color o baja en rendimiento. Aunque no se puede llegar a conclusiones definitivas en relación con los efectos que pudieron tener las infestaciones del ácaro en los rendimientos debido a que las características inherentes de cada una de las variedades pueden influir considerablemente en ello.

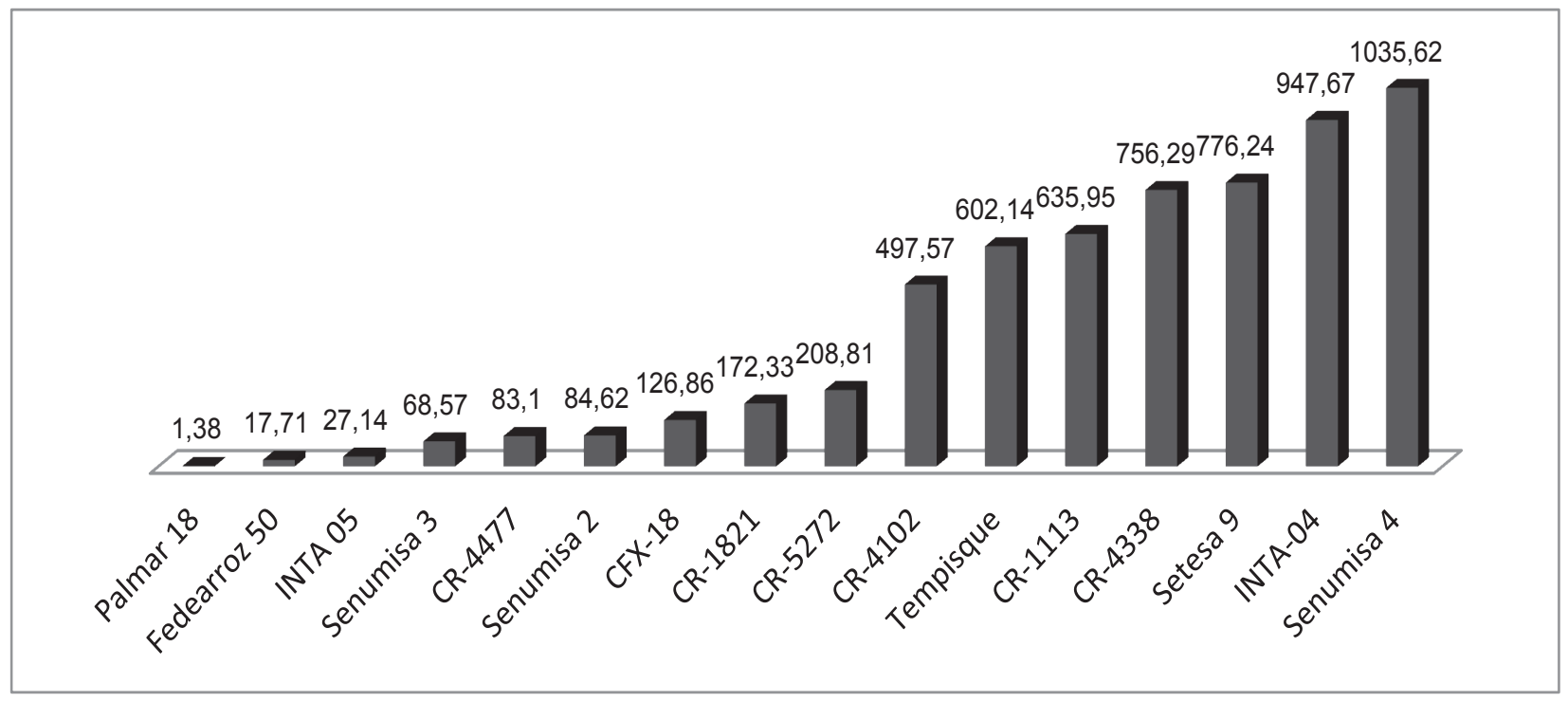

Figura 5. Población total de ácaros de $\mathcal{S}$. spinki por variedad. Palmar Norte, CR. 2005.

\section{ÍNDICE DE PRODUCCIÓN}

Los rendimientos de las diferentes variedades se muestran en el Cuadro 3, se presenta el promedio en gramos producidos en $30 \mathrm{~m}^{2}$, así como el total de la población en 24 plantas evaluadas por variedad. Estos rendimientos se pudieron ver afectados debido a que las variedades desarrollaron su etapa fenológica en distintos momentos y las condiciones de clima variaron en el tiempo. 
Cuadro 3. Duración del ciclo de producción de 16 variedades de arroz y ataque de Steneotarsonemus spinki. Palmar Norte, CR. 2005.

\begin{tabular}{|c|c|c|c|c|}
\hline $\begin{array}{l}\text { Grupo de } \\
\text { susceptibilidad }\end{array}$ & Variedad & $\begin{array}{l}\text { C i c l o } \\
\text { (días) }\end{array}$ & $\begin{array}{c}\text { Infestación promedio } \\
(\%)\end{array}$ & $\begin{array}{c}\text { Rendimiento promedio (gr/ } \\
\text { peso de } 1000 \text { granos) }\end{array}$ \\
\hline \multirow[t]{6}{*}{1} & $\begin{array}{l}\text { Palmar } 18 \\
\text { (Senumisa } \\
\text { Hunsa) }\end{array}$ & 115 & 1,38 & 17,8 \\
\hline & Senumisa 2 & 115 & 84,62 & 15,0 \\
\hline & Senumisa 3 & 115 & 68,57 & 11,4 \\
\hline & INTA 05 & 120 & 27,14 & 17,8 \\
\hline & CR-4477 & 120 & 83,1 & 14,4 \\
\hline & Fedearroz 50 & 128 & 17,71 & 14,9 \\
\hline \multirow[t]{3}{*}{ II } & CFX-18 & 100 & 126,86 & 18,9 \\
\hline & CR-1821 & 130 & 172,33 & 13,2 \\
\hline & CR-5272 & 115 & 208,81 & 22,4 \\
\hline \multirow[t]{7}{*}{ III } & CR-4102 & 110 & 497,57 & 17,8 \\
\hline & Setesa 9 & 128 & 776,24 & 16,7 \\
\hline & Tempisque & 115 & 602,14 & 14,6 \\
\hline & INTA-04 & 115 & 947,67 & 14,4 \\
\hline & CR-1113 & 135 & 635,95 & 14,3 \\
\hline & Senumisa 4 & 118 & 1035,62 & 13,9 \\
\hline & CR-4338 & 115 & 756,29 & 14,1 \\
\hline
\end{tabular}

En la Figura 6 se puede observar que la variedad CR-5272 fue la que mostró el mayor peso de 1000 granos, es posible que esta variable se vea favorecida por la precocidad al igual que CFX-18 seguida de Palmar 18 y CR-4102. Si relacionamos la infestación de ácaros en estas variables, se encuentran en el grupo 2 que corresponde a variedades medianamente preferidas y si continuamos analizando esta comparación no hay claramente relación entre el rendimiento y la densidad poblacional. Sin embargo es una realidad que las variedades producen aún en la presencia de los ácaros, pero se aprecia una ligera tendencia a que en las variedades con más ácaros producen menos con respecto a uno de los componentes de rendimiento promedio.

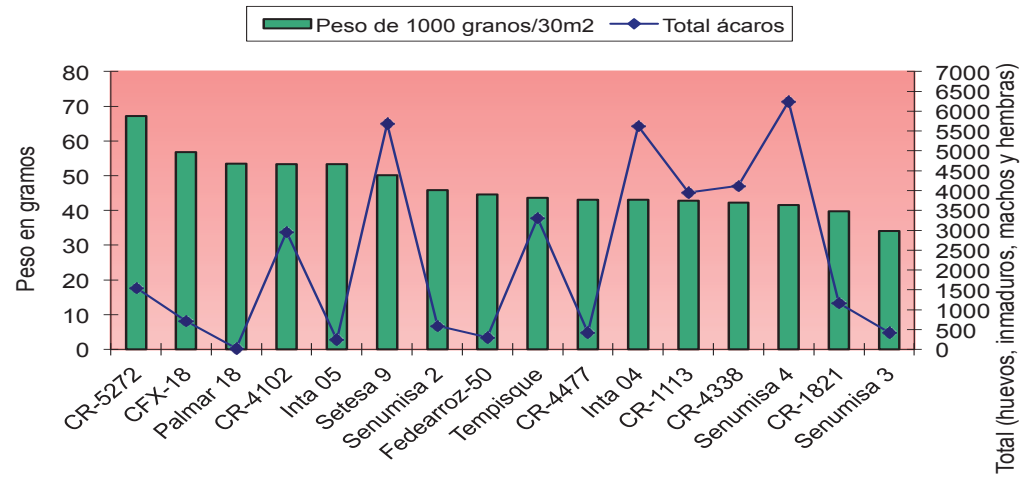

Figura 6. Relación del peso de las variedades con la densidad poblacional de S. spinki. Palmar Norte, CR. 2005. 
Todas las variedades evaluadas son de ciclo precoz a intermedio, ambiente que pudo influir en que no existiera afectación en el rendimiento de las variedades, al no darle tiempo al ácaro de reinfestar la planta en reiteradas ocasiones ya que las variedades de ciclo precoz durante su ciclo de vida logran adquirir menos presión de la población de ácaros con respecto a las variedades tardías, siendo esto de alguna manera un factor que favorece los rendimientos al no dañar las plantas.

En el Cuadro 2, se describen algunas características de las variedades evaluadas en este estudio, para tratar de analizar la preferencia de los ácaros a las diferentes variedades enfocándonos en la conformación del tallo con el fin de constatar si hay algún mecanismo meramente anatómico que favorezca las poblaciones del ácaro, sin embargo es muy escasa la información de este tipo como para poder concluir, basados en las estructuras morfológicas.

Cada variedad evaluada tiene designación y progenitores distintos, por lo que fue muy difícil relacionar las preferencias o no del ácaro con los materiales. No se pudo interpretar algún aporte de gen tolerante o resistente al problema del ácaro.

Pero sí es necesario mencionar que hay variedades que se sacan del mercado debido a la preferencia del ácaro, sin embargo lo importante es valorar si ese material se ve afectado en rendimiento aún en presencia del ácaro. Como ejemplo la variedad CR5272, la cual muestra cierto grado de no preferencia por el ácaro, y a pesar de que son variedades viejas siguen siendo demandadas por los productores debido a su calidad industrial y culinario, al igual que la variedad CR-1113 es muy preferida por el ácaro, pero no se ha demostrado que se disminuya el rendimiento debido a la infestación, se asocia a vaneamiento por hongos o bacterias, pero no por el ácaro.

Este estudio revela que las variedades de arroz toleraron diferentes poblaciones de ácaros por planta, en términos generales se observó que a pesar de que las variedades mantuvieron ácaros en las plantas, éstas produjeron. La población de Steneotarsonemus spinki fue mayor en la etapa de formación de la panícula, declinando posteriormente en la etapa de maduración de la planta. No se presentó una contundente relación entre las poblaciones del ácaro y los rendimientos del cultivo, pero sí en las variedades en que los picos poblacionales no coincidieron con la etapa de formación de la panícula, los rendimientos no se vieron afectados. Hay plantas de arroz de material genético que no deben dejarse de sembrar por el solo hecho de tener altas poblaciones del ácaro, lo que hay que analizar es la etapa fenológica en que alcanza estas poblaciones que es lo que hace que se afecten los rendimientos. Por lo tanto la pérdida en rendimiento con las densidades poblacionales del ácaro es varietal. Es recomendable que en futuros trabajos de este tipo se realicen haciendo coincidir la etapa de floración en todas las variedades.

\section{AGRADECIMIENTOS}

A Carmen Hernández Ramírez, por la asistencia del ensayo y conteo de los ácaros. A Luís Guillermo Vargas Cartagena, por la ayuda en campo, el análisis estadístico y elaboración de gráficos. A Juan Carlos Ocampo Sanabria, por la instalación y manejo agronómico del cultivo. A Luís Diego Riggioni A, por el permiso de realizar este estudio y consecución de las semillas. A Carlos Sanabria Ujueta, por la logística inicial de realizar este estudio. A la MSc. Evelyn Itzel Quirós del IDIAP de Panamá. Penonomé Coclé, por sus consejos y valiosos aportes en la revisión de este estudio.

\section{Literatura consultada}

Almaguel, L.; Santos, A.; Torre, P.; Botta, E.; Hernández, J. 2003. Dinámica de población e indicadores ecológicos del ácaro Steneotarsonemus spinki Smiley 1968 (Acari:Tarsonemidae) en arroz de riego en Cuba. Fitosanidad. 7 (1): 123-130. 
Almaguel, L.; De la Torre, P.; Cáceres, I. 2004. Suma de temperaturas efectivas y potencial de multiplicación del ácaro del vaneado del arroz (Steneotarsonemus spinki, Smiley) en Cuba. Fitosanidad. 8 (1): 37-40.

Botta, E.; Almaguel, L.; González, T.; Arteaga, I. y Hernández, J. 2003. Evaluación del comportamiento de Steneotarsonemus spinki en diferentes variedades de arroz durante los años 2000-2001. Fitosanidad. 7 (2): 125-129.

Cheng, Ch.; Hsiao, C. 1979. Bionomics of $\mathcal{S}$. spinkiattacking rice plants in Taiwan. Recent advances in acaralogy. no. I:111-117.

Cochran, G.; Cox, M. 1965. Diseños experimentales. 2 ed. México. Editorial F. Trillas. p. 618-644.

\section{CONARROZ (Corporación Arrocera} Nacional, Costa Rica). 2009. Estadísticas arroceras. (en línea) Consultado martes 10 de nov. 2009. Disponible en www.conarroz. com.

Hummela, N; Castro, B; McDonald, C; Pellerano, C; Ochoa, R. 2009. The panicle rice mite, Steneotarsonemus spinki Smiley, a re-discovered pest of rice in the United States. Crop Protection 28: 547-560.

IDIAP (Instituto de Investigación Agropecuaria de Panamá). 2006. Guía técnica para el manejo integrado del complejo ácaro-hongobacteria en el cultivo de arroz. $38 \mathrm{p}$.

León, R. 2004. Evaluación de la presencia del ácaro Steneotarsonemus spinki (Tarsonemidae) en diez variedades de arroz (Oryza sativa). INTA-Costa Rica (Archivos técnicos) San José, Costa Rica. 6 p.

León, R. 2008. Evaluación preliminar de diferentes germoplasmas de arroz (Oryza sativa) para determinar la preferencia del ácaro Steneotarsonemus spinki (Tarsonemidae) en Costa Rica. Revista Alcances Tecnológicos. no.1:27-44.
León, R. 2004. Diagnóstico de artrópodos asociados al cultivo de arroz. INTA-Costa Rica. Datos sin publicar

León, R. 2008. Diagnóstico de artrópodos asociados al cultivo de arroz. INTA-Costa Rica. Datos sin publicar

Miranda, I; Ramos, M.; Fernández, B. 2003. Factores que influyen en la abundancia de Steneotarsonemus spinkien arroz, en Cuba. Manejo Integrado de Plagas y Agroecología. no. $69: 34-37$.

INTA (Instituto Nacional de Innovación y Transferencia en Tecnología Agropecuaria); ONS (Oficina Nacional de Semillas). 2005. Producción y certificación de semillas genéticas y fundación de arroz. Estación Experimental EJN, Guanacaste. CR. Boletín divulgativo.

Ramos, M. Y Rodríguez, H. 2003. Aspectos biológicos de Steneotarsonemus spinki en arroz, en Cuba. Manejo Integrado de Plagas y Agroecología. Turrialba, Costa Rica. no. 61: 48-52.

Ramos, M.; Rodríguez, D. J. 2003. Análisis de riesgo de un especie exótica invasora: Steneotarsonemus spinki Smiley, estudio de un caso. Rev. Protección Vegetal. 18(3): 153158.

Reyes, A. 2005. Ácaro del vaneamiento de arroz Steneotarsonemus spinki Smiley (Prostigmata: Tarsonemidae). Programa de arroz. (en línea). CIAT, Colombia. Disponible en www.scribd.com/doc/99235600/AcarosDel-Arroz.

Santos, A.; Almaguel, L.; De La Torre, P.; Cásares, I. 2001. Longevidad y Fecundidad de Steneotarsonemus spinki Smiley (Acari: Tarsonemidae) en el cultivo de arroz en Cuba. Fitosanidad 5 (3): 17-19. 